\title{
Beam Broadening Measured in Transmission Mode at Low Electron Energies in a Scanning Electron Microscope
}

\author{
Milena Hugenschmidt ${ }^{1}$, Erich Müller ${ }^{*}$ and Dagmar Gerthsen ${ }^{1}$ \\ ${ }^{1 .}$ Laboratory for Electron Microscopy, Karlsruhe Institute of Technology, Karlsruhe, Germany. \\ * Corresponding author: erich.mueller@kit.edu
}

In the last years there has been an increasing interest in scanning transmission electron microscopy (STEM) at low primary electron energies $E_{0} \leq 30 \mathrm{keV}$. Low-kV STEM provides high material contrast for materials with low atomic numbers and reduction of the knock-on-damage compared to higher electron energies. Moreover, it can easily be performed in a standard scanning electron microscope, using an annular STEM detector positioned below the sample. However, the mean free path length decreases with lower energies and therefore more scattering events take place. This leads to beam broadening by plural and multiple scattering even for small sample thicknesses, worsening the lateral resolution. Beam broadening in the sample cannot be directly imaged but Drees et al. [1] recently proposed a new technique to measure the width of the transmitted beam. In the present study, we use this approach to analyze beam broadening $b$ in wedge-shaped samples prepared by focused-ion-beam milling of different materials with atomic numbers $Z$ between 10 and 32 for thicknesses up to $900 \mathrm{~nm}$.

A semiconductor STEM detector in a Thermo Fisher DualBeam Strata 400S was used for measuring the transmitted intensity. The STEM detector is partitioned into a bright-field segment, four annular darkfield and a high-angle annular dark-field segment (Fig. 1a). The segments are separately controllable and correspond to specific scattering angle ranges. The measured STEM intensity is normalized with respect to the intensity of the incident electrons after subtracting the back-level intensity of the inactive detector areas. Beam broadening was investigated in materials with known composition $\left(\mathrm{MgO}, \mathrm{Si}_{1} \mathrm{SrTiO}_{3}, \mathrm{Ge}\right)$.

The samples were imaged with distinct detector segments for sample thicknesses $t$ up to $900 \mathrm{~nm}$. Then the normalized intensity $I(t)$ was integrated up to specific angles defined by the number of used detector segments (Fig. 1b). We define $b$ as the beam diameter, which contains $68 \%$ of the total probe intensity neglecting the width of the primary beam which is small compared to $b$. A horizontal line in Fig. $1 \mathrm{~b}$ indicates the thickness where the measured intensity corresponds to $68 \%$. Beam broadening is calculated by $b(t)=t \tan \theta$ with the scattering angle $\theta$, assuming that the electrons are scattered on the average at $t / 2$ as indicated in Fig. 1c. We note that $t$ is known due to the well-defined thickness profile of the wedgeshaped sample. The measured beam broadening is compared with the prediction of Gauvin [2] by fitting the Hurst exponent $\mathrm{H}$ in $b \cdot E_{0}^{(2 H+1) / 2}=(0.1167)^{1-2 H}(39437)^{H} \sqrt{\frac{R}{1-R}} Z^{(4 H+1) / 3}\left(\frac{\rho}{A}\right)^{H} t^{1+H}$ with the amount $R=0.68$ of electrons contributing for the beam diameter, the sample thickness $t$, the atomic mass $A$ and the density $\rho$ of the material. $H$ characterizes different scattering regimes in dependence of the number of scattering events. For all investigated materials and electron energies $E_{0}$ between $15 \mathrm{keV}$ and $30 \mathrm{keV}$ beam broadening can be well described by the equation above with $H=0.75$. This value lies between $H=0.5$ for diffusion regime and $H=1$ for the ballistic regime and is consistent with our definition of the beam diameter and the analyzed energy and thickness range. 
Fig. 2a shows for $E_{0}=25 \mathrm{keV}$ and all investigated materials an increase of $b$ with increasing material density and (average) atomic number as expected for decreasing mean free path lengths. Fig. $2 b$ comprises beam broadening for different primary electron energies $E_{0}$ and materials and shows good agreement for the product $\frac{b E_{0}^{1.25}}{c_{\text {mat }}}$ with $c_{\text {mat }}=\left(\frac{\rho}{A}\right)^{0.75}$ for measured data and the theoretical prediction by Gauvin and Rudinsky [2].

References:

[1] H Drees et al., Ultramicroscopy, 185 (2017), p. 65.

[2] R Gauvin, S Rudinsky, Ultramicroscopy, 167 (2016), p. 21.

[3] The authors acknowledge funding by the Deutsche Forschungsgemeinschaft (DFG) under contract number Ge 841/20-2.

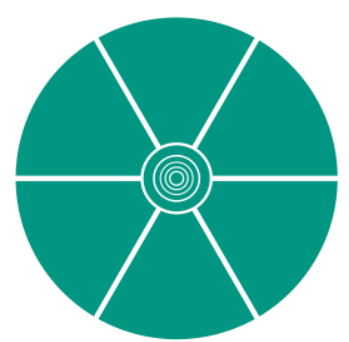

a)

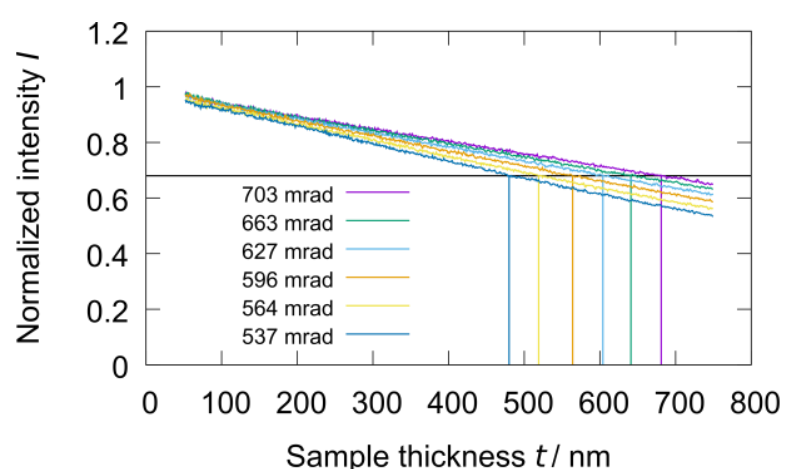

b)

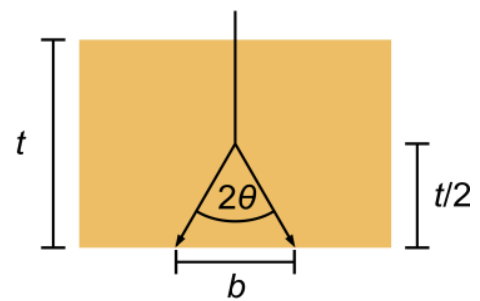

c)

Figure 1. (a) Scheme of the segmented STEM detector. (b) Normalized intensities for Si at $25 \mathrm{keV}$ as a function of the sample thickness and integrated intensities up to the angles indicated in the legend. (c) Calculation of $b$ in a sample of thickness $t$.

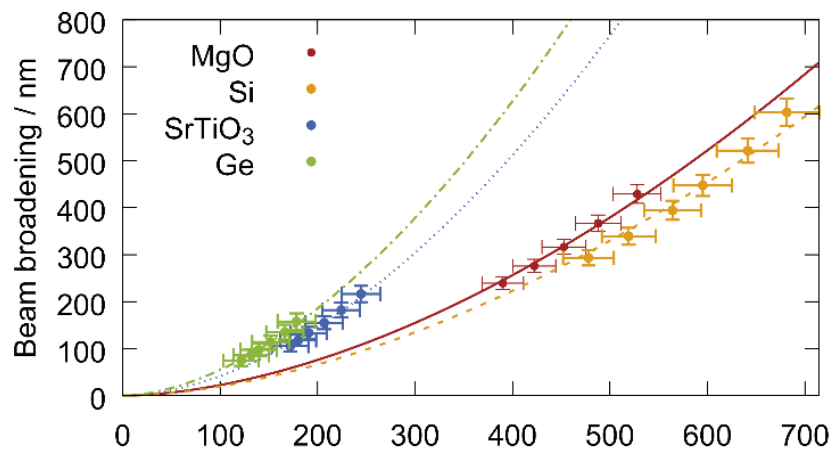

a)

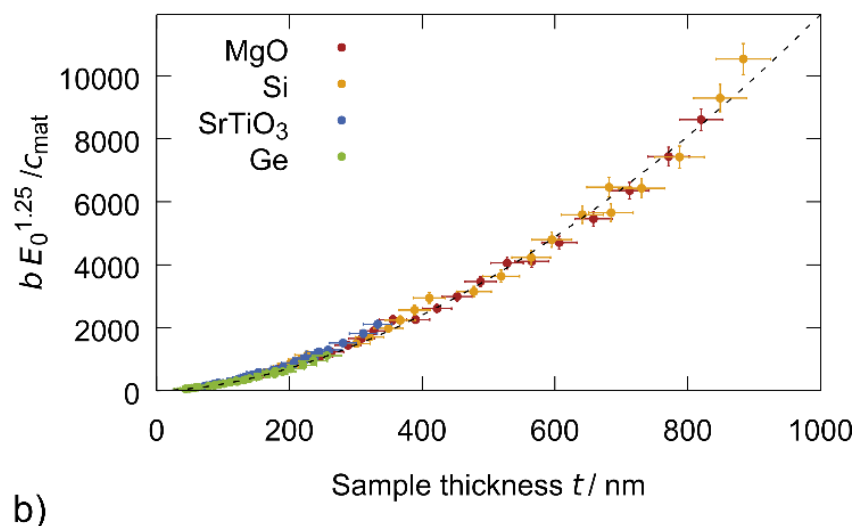

Figure 2. (a) Measured and fitted beam broadening for $E_{0}=25 \mathrm{keV}$ and different materials. (b) Measurements and calculated product $\frac{b E_{0}^{1.25}}{c_{\text {mat }}}$ for different $E_{0}$ and different materials with $c_{\text {mat }}=\left(\frac{\rho}{A}\right)^{0.75}$ as a function of sample thickness. 